\title{
Pediatric Meningiomas- A Case Report and Review of the Literature
}

\author{
Adrian Kelly ${ }^{1 *}$, Tshepang Tau ${ }^{1}$, Tshilidzi Sidiki ${ }^{1}$ and Patrick Lekgwara ${ }^{2}$ \\ ${ }^{1}$ Dr. George Mukhari Academic Hospital, Sefako Makgatho Health Sciences University, South Africa \\ ${ }^{2}$ Department of Neurosurgery, Dr. George Mukhari Academic Hospital, Sefako Makgatho Health Sciences University, South Africa
}

*Corresponding author: Adrian Kelly, Dr. George Mukhari Academic Hospital, FC Neurosurgery (SA), Sefako Makgatho Health Sciences University, 3111 Setlogelo Drive, Ga-Rankuwa Unit 2, Ga-Rankuwa, Pretoria, South Africa, 0208

Submission: August 22, 2018; Published:

\begin{abstract}
Background: Pediatric meningiomas are relatively rare accounting for only $3 \%$ of intracranial neoplasms. As such most of the literature on pediatric meningiomas exists in the form of isolated case reports or as a case series with small patient numbers. Despite this scarcity of literature several important features distinguish pediatric meningiomas from those that occur in adults. These include unique risk factors; a male predominance; larger tumor sizes at presentation; unusual sites of occurrence including intraventricular and parenchymal; higher World Health Organization histological grades; more aggressive clinical behavior and an increased tendency for recurrence. We present a 12-year-old male patient whom presented to our unit with a left fronto-parietal convexity meningioma of an alarming size.
\end{abstract}

Methods: A 12-year-old male patient presented to our unit with an eight-month history of a progressive headache complicated by a two-week history of vomiting. He also complained of progressive weakness of the right side of his body which had worsened to a point that he was no longer independently ambulant. General examination revealed craniomegaly with tortuous scalp veins. Neurological examination revealed a right upper motor neuron facial nerve palsy and a right hemiplegia of $2 / 5$. Radiological investigation revealed an extremely large left fronto-parietal dural based space-occupying lesion which crossed the midline. Due to the adolescent's progressive neurology he was taken to the operating room for emergency resection of the lesion. Gross total resection was achieved, and histopathological analysis confirmed the lesion to be a fibroblastic meningioma.

Result: Post-operatively the patient demonstrated a complete resolution of his hemiplegia and at three weeks post operatively he was already independently ambulant with power $4 / 5$ on the previously hemiplegic side.

Conclusion: Despite the notorious features that characterize pediatric meningiomas, as well as an often-intimidating radiological appearance, adherence to standard Neurosurgical operating principles has the best chance of ensuring a successful outcome.

Keywords: Pediatric meningioma; Benign meningioma pediatrics; Unique features of pediatric meningiomas

\section{Introduction}

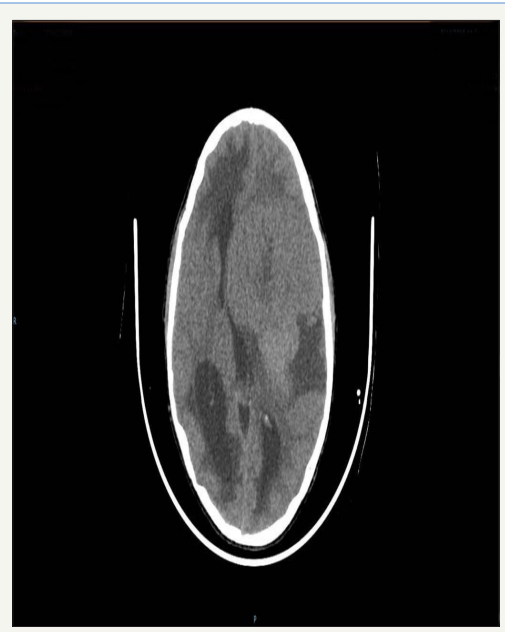

Figure 1: Pre-operative axial CT brain: showing left frontoparietal isodense space occupying lesion with entrapment hydrocephalus of right lateral ventricle.
Meningiomas arise from arachnoid cap cells and in adults comprise $20-30 \%$ of primary intracranial neoplasms (Figure 1). In the pediatric population they are however relatively rare and comprise between 1 and 3\% of primary intracranial neoplasms $[1,2]$. While having a female predominance in the adult population in the pediatric population several studies note these tumors to occur more commonly in males $[3,4]$. The male to female ratio in the pediatric population is described to be as high as $2-3: 1[5,6]$.

Unique risk factors exist for the development of meningiomas in the pediatric population and the strongest association is in patients with neurofibromatosis type II where a finding of multiple intracranial meningiomas is not uncommon. In fact, between 6-39\% of pediatric meningiomas are reported to occur in children with neurofibromatosis type II [7]. Another study reported meningiomas to occur in $53 \%$ of neurofibromatosis type II patients and in the most severe phenotype called the Wishart variant meningiomas occur in up to $83 \%$ of patients [8]. The next strongest 
association regarding pediatric meningiomas is in children whom have had previous ionizing radiation exposure $[9,10]$. Here the latency period is described to be on average 25 years in a study that evaluated the meningioma incidence in patients irradiated for childhood leukemia [11]. This specific subset of radiation induced meningiomas behave quite differently from sporadic meningiomas and are more often multifocal, more aggressive and exhibit a higher recurrence rate [12]. Besides the two established risk factors for pediatric meningiomas mentioned above several others are noted, although not specific for pediatric meningiomas but rather meningiomas in general, and include a family history of meningiomas, hormonal factors, obesity and cigarette smoking [1316].

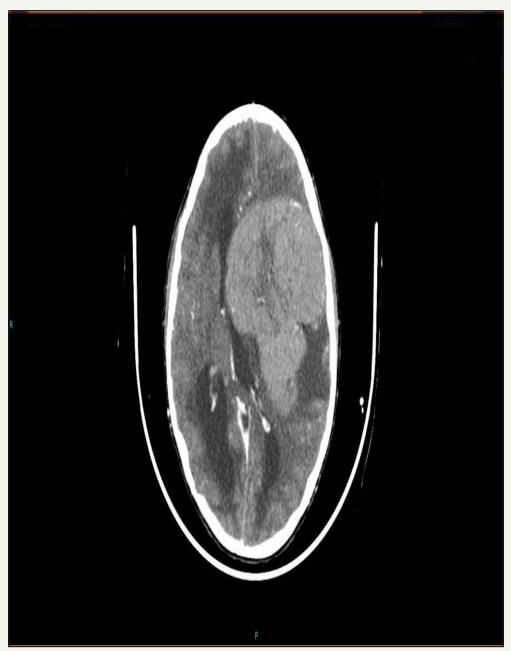

Figure 2: Pre-operative axial CT brain post contrast: shows the heterogeneous enhancement of the same lesion.

At a histological level the most common types of pediatric meningiomas are, like in adults, the meningothelial and fibroblastic subtypes [17]. When considering pediatric meningiomas overall several studies do however note a proportionately higher percentage of WHO grade II and III tumors (Figure 2). In pediatric meningiomas the mitotic rates as well as their proliferation indices are commonly higher, and they exhibit an increased rate of brain invasion as compared to the typical meningiomas that occur in adults $[12,18]$. They are also reported in several studies to exhibit aggressive behavior and to have an increased recurrence rate [1921].

The unusual topographical distribution of pediatric meningiomas also sets them apart from their adult counterparts. Like adults the cerebral convexities have been reported to be the most common location for pediatric meningiomas in some series (Figure 3). Other studies note an increased occurrence of pediatric meningiomas in unusual locations such as intraventricular, parenchymal and in the cerebellopontine angle [22-25]. Other sites noted to predominate in the pediatric population are the orbit and optic nerve sheath and in contrast to adults' certain sites are extremely rare in children which include the suprasellar and parasellar regions, the cavernous sinus and around the foramen magnum [26].

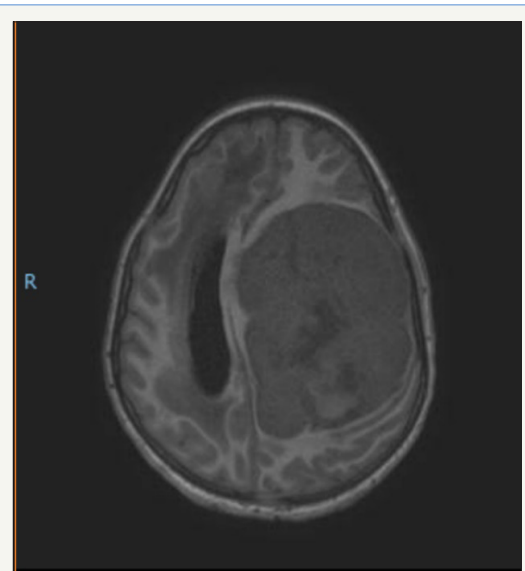

Figure 3: Pre-operative axial T1W MRI: shows hypo-intense lesion as described.

\section{Methods}

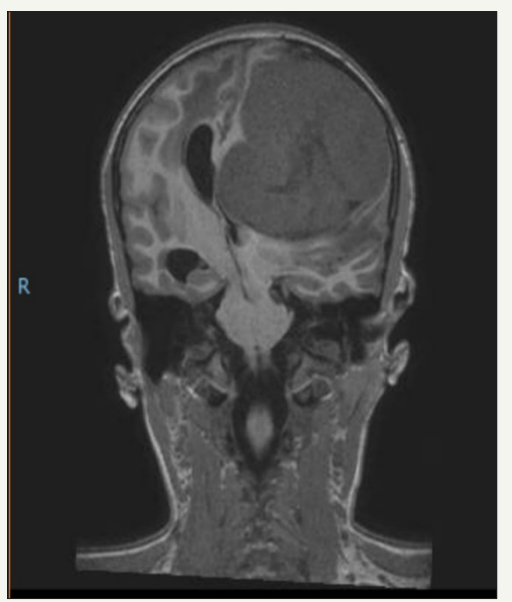

Figure 4: Pre-operative T1W coronal MRI: showing dural based nature of the lesion and subfalcine herniation.

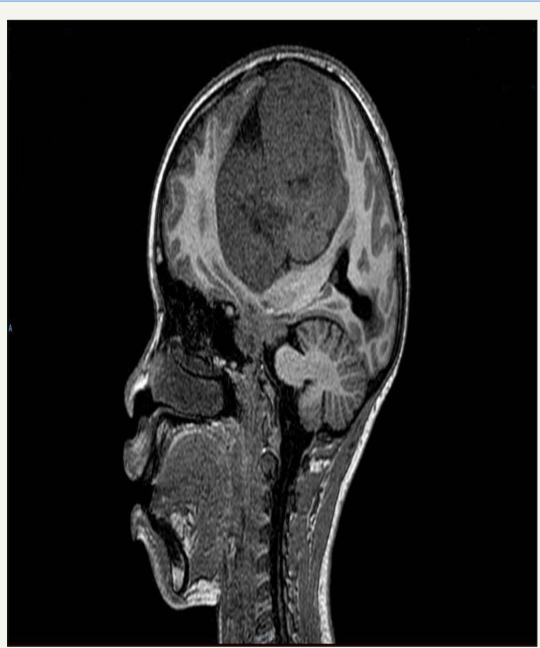

Figure 5: T1W sagittal MRI: showing enormous size of this lesion extending from the vertex to the skull base.

A 12-year-old male patient presented to our unit with an eightmonth history of a progressive headache complicated by a twoweek history of blurring of vision and vomiting (Figure 4). He also 
complained of progressive weakness of the right side of his body of 3 months duration which had recently worsened to a point that he was no longer independently ambulant. General examination revealed craniomegaly with an occipito-frontal circumference of $45 \mathrm{~cm}$ and tortuous scalp veins. Neurological examination revealed the patient to be fully conscious and orientated to time person and place. Cranial nerve examination revealed a right upper motor neuron facial nerve palsy and motor examination revealed a right hemiplegia of 2/5 in all myotomes (Figure 5).

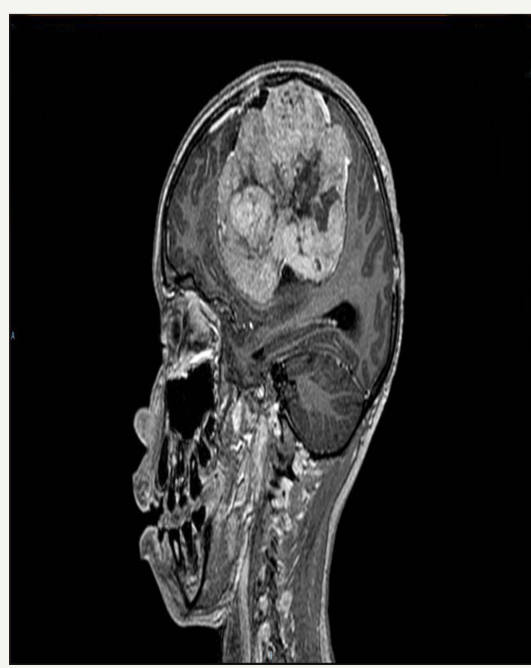

Figure 6: MRI T1W post contrast: Shows heterogenous enhancement pattern.

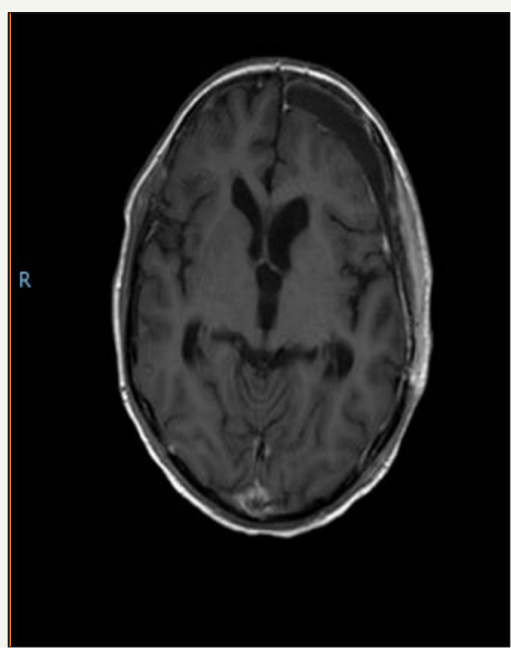

Figure 7: Post -operative $\mathrm{T} 1 \mathrm{~W}$ axial: showing gross total resection.

A computed tomographic brain scan was performed which revealed an isodense well-defined dural based left fronto-parietal space occupying lesion that on the coronal view demonstrated subfalcine herniation (Figure 6). The lesion measured $87 \times 81 \times 90 \mathrm{~mm}$ and entrapment hydrocephalus of the right lateral ventricle was present. With the administration of contrast the lesion showed avid heterogeneous enhancement. An MRI showed the lesion to be well demarcated and hypo intense on T1 weighted imaging and hyper intense on $\mathrm{T}_{2}$ weighted imaging (Figure 7). With the administration of gadolinium, the lesion showed heterogeneous enhancement.
MR spectroscopy was performed which noted a distinctive choline peak.

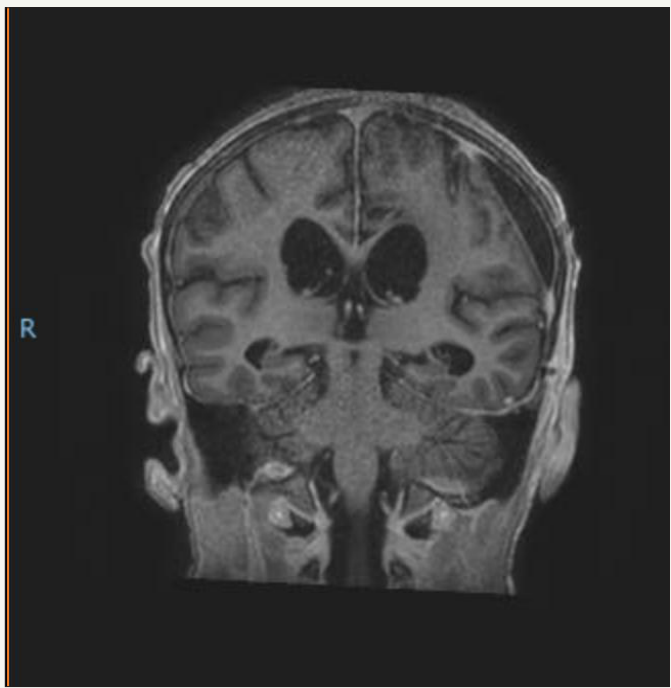

Figure 8: Post-operative T1W coronal: showing gross total resection.

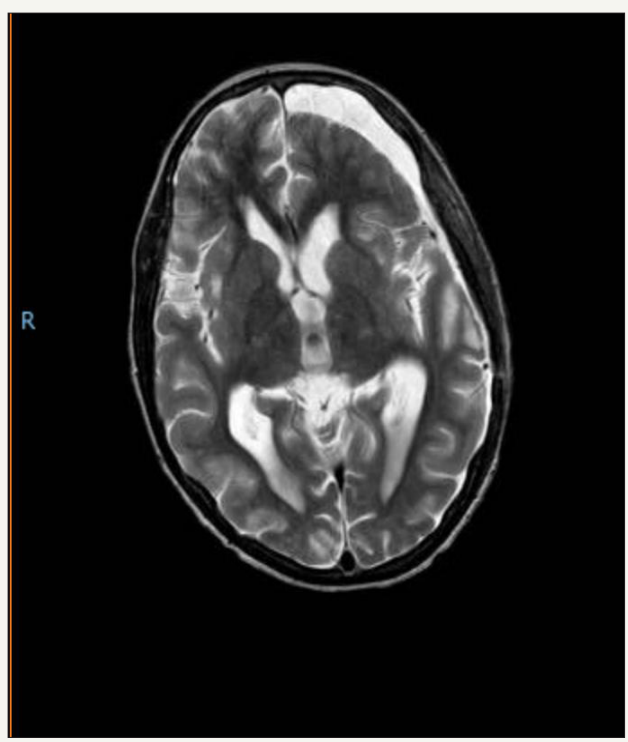

Figure 9: Post-operative T2 axial: showing cerebrospinal fluid adjacent to the re-expanded brain and a small resection cavity.

Due to the adolescent's progressive neurology he was taken to the operating room for emergency micro-surgical resection of the lesion (Figure 8). A left fronto-parietal craniotomy was performed, and the lesion was noted to be dural based with no obvious overlying bony involvement. The tumor consistency was firm and after devascularising the tumor by excising its dural base demonstrated moderate bleeding while being internally debulked. The tumor rim separated easily from the surrounding brain tissue and was removed (Figure 9). A duroplasty was performed using a synthetic dural substitute and the bone replaced and reattached with mini-plates and screws. The scalp was closed in layers and the patient was transferred to the Intensive Care Unit (Figure 10). 


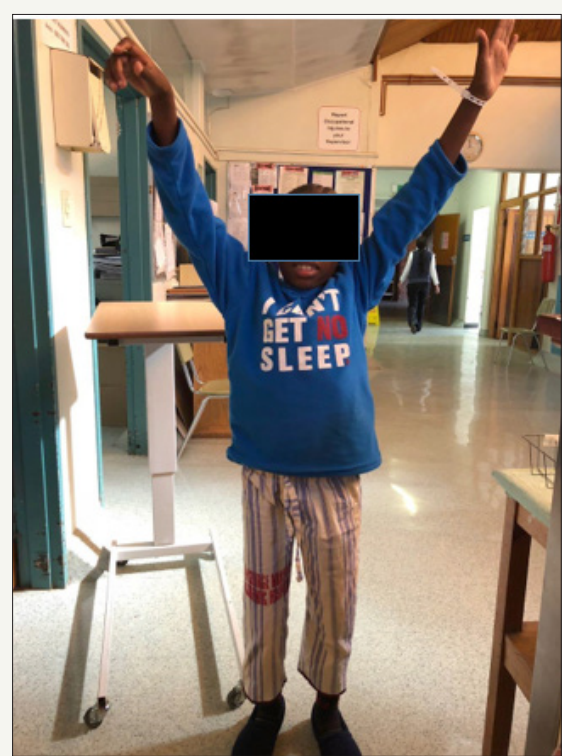

Figure 10: Post-operative photo: At 3 weeks post operatively, the right hemiplegia 2/5 has resolved to a hemiparesis $4 / 5$. This photo demonstrates the improvement in power of the upper limb.

\section{Result}

The child was extubated on day two post-operatively and a post-operative MRI confirmed that gross total resection had been achieved. Histopathological analysis confirmed the lesion to be a fibroblastic meningioma. Over the subsequent two weeks the child demonstrated a gradual resolution of his right hemiplegia. At three weeks post-operatively he was already independently ambulant with power $4 / 5$ on the previously hemiplegic side. He was discharged into the care of his parents and booked for out-patient review in six months. No adjuvant radiotherapy was planned.

\section{Discussion}

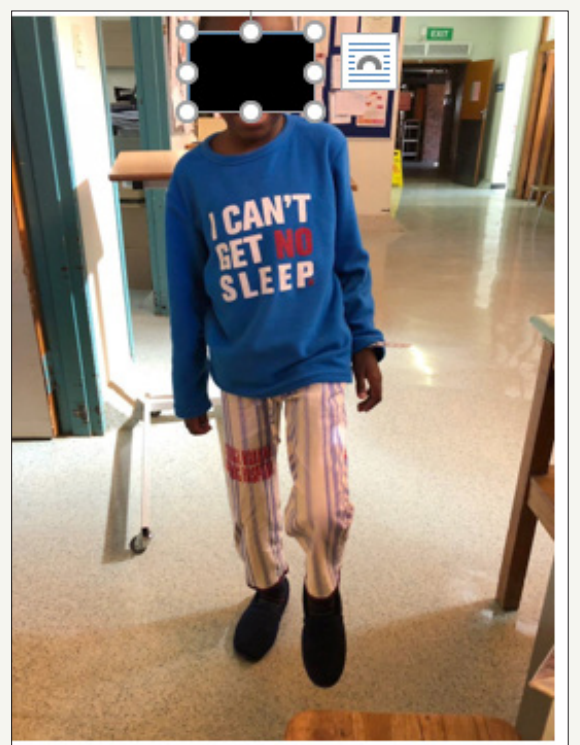

Figure 11: Post-operative photo: At 3 weeks post operatively, the right hemiplegia $2 / 5$ has resolved to a hemiparesis $4 / 5$. This photo demonstrates the child weight bearing on the lower limb.

Our patient had no family history of Neurofibromatosis nor previous exposure to ionizing radiation and hence this pediatric meningioma would be regarded as sporadic. The craniomegaly suggests a slow growing tumor which over time was partially accommodated by a disproportionately enlarging calvarium. The slow growing nature of this meningioma is also supported by the fact that despite its enormous size the child was fully conscious due to the brain having time to compensate for the slowly enlarging mass. This is further supported by the histopathological result of a fibroblastic meningioma which is a WHO grade I variant and considered benign (Figure 11). 
Regarding prognosis a large meta-analysis recently published noted a $12 \%$ overall mortality for pediatric meningiomas of which $25 \%$ are secondary to intra-operative complications [26]. Another study noted an intra-operative complication rate of $46 \%$ in pediatric patients undergoing meningioma resection procedures, with the result that these patients often have long hospital stays [27]. Deaths related to post-operative adjuvant radiotherapy are also recognized and add to the mortality rate [26].

Due to our patient having experienced a successful gross total resection confirmed by post-operative MRI as well as the benign histopathological result, adjuvant radiotherapy and the potential complications of this were avoided. Re-operation rather than radiation would be considered as the first line therapy for recurrence in our particular patient.

In conclusion pediatric meningiomas are a specific neurosurgical consideration and should not be seen as on a par with the adult form of the disease. Besides the pediatric meningioma itself special surgical considerations related to the pediatric patient undergoing cranial surgery must also be considered, and planned for, to ensure a successful operative outcome [28]. These tumors require an interdisciplinary team for a successful outcome and as our case demonstrates this can be achieved.

\section{Conflict of Interests}

None of the authors listed below have any financial nor personal relationships with other people, or organizations, that could inappropriately influence (bias) their work, all within 3 years of the beginning the work submitted.

\section{Funding}

This research did not receive any specific grant from funding agencies in the public, commercial, or not-for-profit sectors.

\section{References}

1. Goshen Y, Stark B, Kornreich L, Michowitz S, Feinmesser M, et al (2007) High incidence of meningioma in cranial irradiated survivors of childhood acute lymphoblastic leukemia. Pediatr Blood Cancer 49(3): 294-297.

2. Baumgartner J, Sorenson J (1996) Meningioma in the pediatric population. J Neuroncol 29(3): 223-228.

3. Ferrante L, Acqui M, Artico M, Mastronardi L, Rocchi G (1989) Cerebral meningiomas in children. Childs Nerv Syst 5(2): 83-86.

4. Germano IM, Edwards M, Davis R, Schiffer D (1994) Intracranial meningiomas of the first two decades of life. J Neurosurg 80(3): 447453.

5. Maranhao P, Campos J, Lima M (2008) Intracranial meningiomas in children: ten-year experience. Pediatr Neurol 39(6): 415-417.

6. Jaiwal S, Vij M, Mehotra A, Jaiswal AK, Srivastava AK (2011) A clinicopathological and neuroradiological study of pediatric meningioma from a single centre. J Clin Neurosci 18(8): 1084-1089.

7. Perry A, Dehner L (2003) Meningeal tumors of childhood and infancy. An update and literature review. Brain Pathol 13(3): 386-408.

8. Evans D, Huson S, Donnai D, Neary W, Blair V, et al. (1992) A clinical study of type 2 neurofibromatosis. Q J Med 84(304): 603-618.
9. Elbabaa S, Gokden M, Crawford J, Kesari S, Saad A (2012) Radiationassociated meningiomas in children: clinical, pathological, and cytogenetic characteristics with a critical review of the literature. J Neurosurg Pediatr 10(4): 281-290.

10. Kotecha R, Pascoe E, Rushing E, Rorke AL, Zwerdling T, et al. (2011) Meningiomas in children and adolescents: a meta-analysis of individual patient data. Lancet 12(13): 1229-1239.

11. Banerjee J, Paakko E, Harila M, Herva R, Tuominen J, et al. (2009) Radiation induced meningiomas: a shadow in the success story of childhood leukaemia. Neuro Oncol 11(5): 543-549.

12. Deen H, Scheithauer B, Ebersold M (1982) Clinical and pathological study of meningiomas of the first two decades of life. J Neurosurg 56(3): 317-322.

13. Claus E, Calvocoressi L, Bondi M, Schildkraut J, Wiemels J, et al. (2011) Family and personal medical history and risk of meningioma. J Neurosurg 115(6): 1072-1077

14. Custer B, Longstreth W, Phillips L, Koepsell T, Van BG (2006) Hormonal exposures and risk of intracranial meningioma in women: a population-based case control study. BMC Cancer 6: 152.

15. Niedermainer T, Behrens G, Schmidt D, Schlecht I, Fischer B, et al. (2015) Body mass index, physical activity, and risk of adult meningioma and glioma: a meta-analysis. Neurology 85(15): 1342-1350.

16. Phillips L, Longstreth W, Koepsell T, Custer B, Kukull W, et al. (2005) Active and passive cigarette smoking and risk of intracranial meningioma. Neuroepidemiology 24(3): 177-122.

17. Maillo A, Orfao A, Espinosa AB, Sayagués JM, Merino M, et al. (2007) Early recurrences in histologically benign/grade I meningiomas are associated with large tumors and coexistence of monosomy 14 and del(1p36) in the ancestral tumor cell clone. Neuro Oncol 9(4): 438446.

18. Perry A, Giannini C, Raghavan R, Scheithauer B, Banerjee R, et al. (2001) Aggressive phenotypic and genotypic features in pediatric and NF-2 associated meningiomas: a clinicopathologic study of 53 cases. J Neuropathol Exp Neurol 60(10): 994-1003.

19. Arivazhagan A, Devi B, Kolluri S, Abraham R, Sampath S, et al. (2008) Pediatric intracranial meningiomas- do they differ from their counterparts in adults? Pediatr Neurosurg 44(1): 43-48.

20. Drake J, Hendrick E, Becker L, Chaung S, Hoffman H, et al. (1985) Intracranial meningiomas in children. Pediatr Neurosci 12(3): 134139.

21. Thuijs N, Uitdehaag B, Van OW, Vandertop W, Peerdeman S, et al. (2012) Pediatric meningiomas in the Netherlands 1974-2010: a descriptive epidemiological case study. Child's Nerv Syst 28(7): 1009-1015.

22. Prabhu VC, Perry EC, Melian E, Barton K, Guo R, et al. (2014) Intracranial meningiomas in individuals under the age of 30; analysis of risk factors, histopathology, and recurrence rate. Neurosci Discov $2: 1$

23. Santos MV, Furlanetti L, Valera ET, Brassesco MS, Tone LG, et al. (2012) Pediatric meningiomas: A single-center experience with 15 consecutive cases and review of the literature. Childs Nerv Syst 28(11): 1887-1896.

24. Kotecha RS, Pascoe EM, Rushing EJ, Rorke ALB, Zwerdling T, et al (2011) Meningiomas in children and adolescents: A meta-analysis of individual patient data. Lancet Oncol 12(13): 1229-1239.

25. Sheikh BY, Siqueira E, Dayel F (1996) Meningioma in children: A report of nine cases and a review of the literature. Surg Neurol 45(4): 328335.

26. Gump WC (2015) Meningiomas of the pediatric skull base: A review. J Neurol Surg B Skull Base 76(1): 66-73. 
27. Kotecha R, Pascoe E, Rushing E, Rorke AL, Zwerdling T, et al. (2011) Meningiomas in children and adolescents: a meta-analysis of individual patient data. Lancet Oncol 12(13): 1229-1239.
28. Gao X, Zhang R, Mao Y, Wang Y (2009) Childhood and juvenile meningiomas. Childs Nerv Syst 25(12): 1571-1580. (c) (i) Creative Commons Attribution 4.0

For possible submissions Click Here
Submit Article

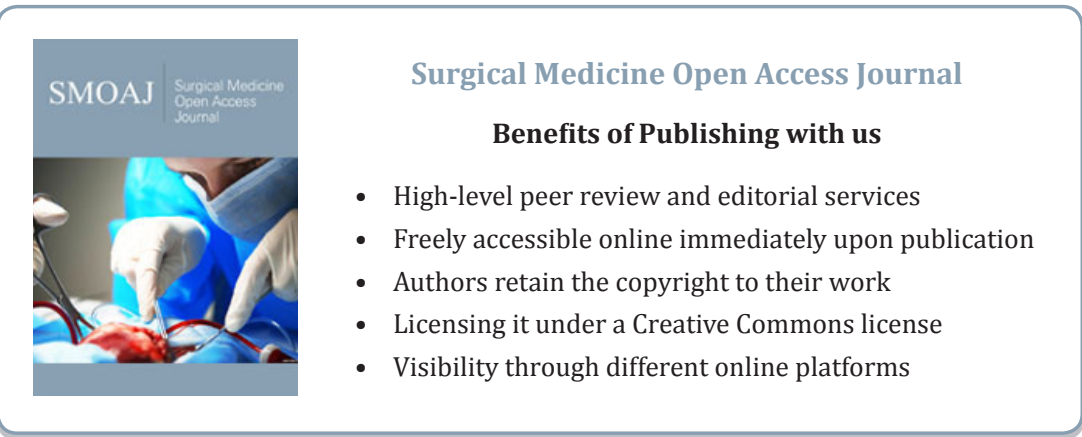

